\title{
Ratchet-like dynamics of fluxons in annular Josephson junctions driven by bi-harmonic microwave fields
}

\author{
A. V. Ustinov, C. Coqui and A. Kemp \\ Physikalisches Institut III, Universität Erlangen-Nürnberg, D-91058 Erlangen, Germany \\ Y. Zolotaryuk \\ Bogolyubov Institute for Theoretical Physics, National Academy of Sciences of Ukraine, Kiev 03143, Ukraine \\ M. Salerno \\ Dipartamento di Fisica "E. R. Caianiello" and Istituto Nazionale di Fisica della Materia (INFM), \\ Universita? di Salerno, I-84081 Baronissi, Salerno, Italy \\ (September 22, 2018) \\ Experimental observation of the unidirectional motion of a topological soliton driven by a bi- \\ harmonic ac force of zero mean is reported. The observation is made by measuring the current- \\ voltage characteristics for a fluxon trapped in an annular Josephson junction that was placed into a \\ microwave field. The measured dependence of the fluxon mean velocity (rectified voltage) at zero dc \\ bias versus the phase shift between the first and second harmonic of the driving force is in qualitative \\ agreement with theoretical expectations. \\ PACS numbers: $05.45 .-\mathrm{a}, 74.50 .+\mathrm{r}, 85.25 .-\mathrm{j}$
}


The role played by solitons in various physical systems is important and commonly known. Experimental observation of dynamical effects produced by solitons is in many cases difficult because real systems are very often far from the idealized mathematical models which give rise to soliton solutions. Solitons of the most common type are topological kinks, a well-known example being a magnetic flux quantum (fluxon) in long Josephson junctions (LJJs) ${ }^{1,2}$. A fluxon in LJJ can be easily driven by the bias current applied to the junction. The motion of a fluxon gives rise to the dc voltage $V$ across the junction, which is proportional to the fluxon's mean velocity. Varying the dc bias current $I$, one can produce a dependence $V(I)$, which is the main dynamical characteristic of the LJJ. An experimentally obtained $V(I)$ curve allows to identify many interesting features in the dynamics of fluxons trapped in a LJJ ${ }^{2}$.

A microwave field applied to a LJJ gives rise to an ac drive acting on the fluxon. In a spatially homogeneous lossy system, an ac drive may only induce an oscillatory motion of a kink, which is hard to observe in LJJ due to the absence of dc voltage. It was first predicted ${ }^{3}$ and then observed experimentally ${ }^{4}$ that an ac drive can support motion of a kink with a nonzero average velocity $\langle v\rangle$ in a system with a periodic spatial modulation. Thus, if an inhomogeneous spatial potential is present, the fluxon motion can be locked to the external ac drive frequency. In this case the vertical steps ("Shapiro steps") appear in the dependence of the average fluxon velocity $\langle v\rangle$ on the dc bias. The fluxon velocity in this frequency-locked state can be either positive or negative, depending on the initial conditions. Another interesting effect for a homogeneous system, in which the ac-driven fluxon drift may occur in either direction but is not frequency locked, was recently reported by Goldobin et al. ${ }^{5}$.

Transport phenomena in nonlinear systems can also be induced by the so called ratchet effect. This phenomenon has been studied in a large variety of physical systems ${ }^{6}$ and manifests itself by an unidirectional motion under the influence of forces with zero mean. The underlying mechanism is the breaking of those spatial and/or temporal symmetries ${ }^{7}$ of the system, which connect trajectories with specular velocities (as well as the possibility of phase-locking of the particle dynamics by the external field ${ }^{8}$ ). So far, great attention has been devoted to the study of the soliton motion in ratchet potentials. Josephson fluxon ratchets have been proposed and designed using either continuous long junctions of special shapes ${ }^{9,10}$ or discrete junction arrays ${ }^{11,12}$, including the quantum case ${ }^{13}$. In these structures, an ac drive applied to a fluxon placed into an asymmetric (ratchet-like) spatial potential leads to the rectified voltage at zero bias. The mechanism underlying soliton ratchets in asymmetric potentials and driven by harmonic forces was investigated for the double-sine-Gordon system which can be mapped to an array of 3 -junction SQUIDs ${ }^{14}$. The possibility of a ratchet-like effect induced by more complex ac drives but in the absence of any spatial ratchet potential was also demonstrated $^{15,16}$.

The importance of the ratchet-like effect induced by a bi-harmonic force in the context of Josephson junctions resides in the fact that it gives a non-zero voltage state in absence of dc bias. The resulting current-voltage characteristics of a uniform LJJ is fully controlled by the phase difference between the two microwave field components driving the junction, which is a nice feature for practical applications. In a more general context, the experimental observation of soliton ratchets induced by asymmetric forces, first reported in this paper, opens new perspective for soliton transport in other physical systems. In contrast to soliton ratchets induced by asymmetric potentials, which are generally more difficult to implement and to control (a change of the potential requires a structural change of the system), soliton ratchets induced by asymmetric external fields can be implemented in any physical system described by soliton equations.

The aim of this Letter is to present the first experimental observation of the rectified dc voltage induced by the unidirectional motion of a fluxon driven by bi-harmonic microwaves of zero average, applied to a spatially uniform long Josephson junction. Our results qualitatively confirm the theoretical predictions ${ }^{15,16}$.

Experiments have been performed using $\mathrm{Nb} / \mathrm{Al}-\mathrm{AlO}_{x} / \mathrm{Nb}$ Josephson junctions ${ }^{17}$. We have measured two samples which have shown similar behavior; here we present data for one of them having the mean diameter $2 R=100 \mu$ m and the annulus width $W=4 \mu \mathrm{m}$. The dc bias current was applied to the ring-shaped junction via the leads having the so-called Lyngby geometry ${ }^{18}$ (schematically shown in Fig. 1). In this configuration the bias electrode width equals the ring diameter, and the bias current is distributed very uniformly around the sample. This is due to the fact that the current distribution carried by a superconducting strip is larger on the edges and its profile $\left(R^{2}-y^{2}\right)^{-1 / 2}($ where $y$ is the transverse coordinate ${ }^{19}$ ) exactly matches the uniform biasing of the junction.

The critical current $I_{\mathrm{c}}$ of this junction in the state with no trapped fluxons was found to be about 0.9 of the maximum expected value, corresponding to a nearly uniform current flow over the junction area. The junction has the critical current density of about $1 \mathrm{kA} / \mathrm{cm}^{2}$, which corresponds to the Josephson length of $\lambda_{\mathrm{J}} \approx 12 \mu \mathrm{m}$ and plasma frequency of about $f_{p}=120 \mathrm{GHz}$. This implies the normalized junction length $2 \pi R / \lambda_{\mathrm{J}} \equiv \ell \approx 26$ and width $W / \lambda_{\mathrm{J}}<1$, i.e. the junction can be regarded as long and quasi-one-dimensional. The measurements were done at the temperature $4.2 \mathrm{~K}$, using a shielded low-noise measurement setup. The radio-frequency (rf) current was supplied by means of an open-ended coaxial cable antenna placed above the junction. The antenna was oriented parallel to the dc bias current leads, therefore the inductively coupled rf current was flowing in parallel to dc current. The applied ac power was supplied by the two microwave sources (HP8672A and HP83620A, combined via a directional coupler) having the frequencies $f_{1}=f=\omega /(2 \pi)$ and $f_{2}=2 f$ with $f$ in the range between 1 and $5 \mathrm{GHz}$. The sources were phase locked 
to a common $10 \mathrm{MHz}$ reference signal. The phase shift $\theta$ between the sources was controlled electronically via the phase of the reference signal and monitored using a network analyzer. The ac power levels $P_{1}$ and $P_{2}$, mentioned below, pertain to the input at the top of the cryostat.

Trapping of a fluxon in the junction was achieved by cooling the sample below the critical temperature $T_{c} \approx 9.2 \mathrm{~K}$ for the transition of $\mathrm{Nb}$ into the superconductive state, with a small dc bias current applied to the junction ${ }^{20}$. The residual fluxon depinning current $I_{\mathrm{dep}}$ was found to be rather small, less than $3 \%$ of the Josephson critical current $I_{\mathrm{c}}$, measured without the trapped fluxon. As a fluxon can only be trapped by junction's local inhomogeneities in the absence of the magnetic field, this indicates a fairly high uniformity of the junction.

In our experimental configuration, the dynamics of the superconducting phase difference across the junction is described by the perturbed sine-Gordon equation

$$
\begin{aligned}
& \varphi_{t t}-\varphi_{x x}+\sin \varphi+\alpha \varphi_{t}=\gamma+\tilde{\gamma}(t) \\
& \tilde{\gamma}(t)=\tilde{\gamma}_{1} \sin (\Omega t)+\tilde{\gamma}_{2} \sin (2 \Omega t+\theta)
\end{aligned}
$$

with the boundary conditions

$$
\varphi(\ell)=\varphi(0)+2 \pi ; \quad \varphi_{x}(\ell)=\varphi_{x}(0),
$$

where $x$ and $t$ are the length along the junction and the time, measured, respectively, in units of the Josephson length $\lambda_{\mathrm{J}}$ and the inverse plasma frequency $\left(2 \pi f_{p}\right)^{-1}$. The dissipation constant due to the quasiparticle tunneling current, $\alpha$, is of the order of 0.05 in our experiment. Here $\gamma, \tilde{\gamma}_{1}$ and $\tilde{\gamma}_{2}$ are the dc and ac bias current densities, both normalized to the junction's critical current density and $\Omega=\omega /\left(2 \pi f_{p}\right)=f / f_{p}$ is the dimensionless frequency of the ac bias. The amplitudes $\tilde{\gamma}_{1} \sim \sqrt{P_{1}}$ and $\tilde{\gamma}_{2} \sim \sqrt{P_{2}}$ correspond to the first and second microwave harmonics, respectively, and $\theta$ is the phase shift between them that we controlled in this experiment.

The effect of a single harmonic microwave (here $\tilde{\gamma}_{2}=0$ ) on the motion of a fluxon in the junction is presented in Fig. 2a. It shows the current-voltage characteristics of the fluxon with no ac drive (open symbols) and with the single-harmonic ac drive (solid symbols) having the frequency $f=4.8 \mathrm{GHz}$. We note that the fluxon characteristics in the presence of a monochromatic ac drive of any frequency remains symmetric with respect to the origin, as was observed in the earlier experiments ${ }^{4,5}$.

The effect of the second harmonic in the microwave spectrum is reported in Fig. 2b. The two curves, shown in that figure, correspond to two different phase shifts between the drives $P_{1}$ and $P_{2}$. The salient feature of these curves is the non-zero voltage at zero dc current through the junction. The sign of this rectified voltage gets reversed by changing the phase shift $\theta$ from $\pi / 2$ to $3 \pi / 2$ (or to $-\pi / 2$ ). The dependence of the rectified voltage at zero bias on the phase shift is presented in Fig. 3(a). We observe that the rectified voltage reverses its sign close to $\theta=0$ and $\theta=\pi$ and changes periodically with the phase shift. We also measured the dependence of this effect on power and found that, at somewhat lower power levels, the dependence of the rectified voltage on the phase shift may have several maxima within period of $2 \pi$, see Fig. $3(\mathrm{~b})$. Possibly, at these values of the ac bias the inhomogeneity of the junction becomes significant, and the ideal model accounted by Eq. (1) is no longer valid. Numerical simulations of Eq. (1) with boundary conditions (2) have been performed with the 4th order Runge-Kutta method. The average voltage drop has been computed and plotted in Figs. $3(\mathrm{a}, \mathrm{b})$ with the solid line. The following experimental values of the systems parameters have been taken: $\alpha=0.05, \Omega=0.01, \tilde{\gamma}_{1}=\tilde{\gamma}_{2}=0.1$ for the case of Fig. 3(a) and $\Omega=0.02$, $\tilde{\gamma}_{1}=\tilde{\gamma}_{2}=0.135$ for the case of Fig. 3(b).

The results of the experimental measurements and numerical simulations are in good correspondence with the results of the first order (point-particle approximation) soliton perturbation theory ${ }^{21}$. In this approximation the perturbation is assumed to be small, so that it does not change the shape of the soliton and only the soliton parameters vary in time. Also, this approximation works well in the adiabatic limit, when $\Omega \ll \alpha$. Using this approach, the mean fluxon velocity (in the absence of dc bias) can be computed ${ }^{16}$ as follows:

$$
\langle v\rangle \sim \tilde{\gamma}_{1}^{2} \tilde{\gamma}_{2} \sin \left(\theta+\theta_{0}\right), \quad \tan \theta_{0}=\frac{2}{\frac{\alpha}{\Omega}\left[3+\left(\frac{\alpha}{\Omega}\right)^{2}\right]} .
$$

The voltage drop is proportional to the average fluxon velocity. Thus, the rectified voltage behaves as a sine function of the phase shift. Note that $\theta_{0} \rightarrow 0$ if $\Omega \rightarrow 0$, and this is in accordance with experimental and numerical data, where the parameter $\theta_{0}$ is small.

The maximum amplitude of the rectified voltage is rather large - it corresponds to the average normalized fluxon velocity of about 0.2 . Figure 4 shows the power dependence of the maximum amplitude of the rectified dc voltage. For each of the two curves the power level of another harmonic was kept constant (but not referenced). One can see that the rectified voltage has a maximum value at some optimum power level. The most important system parameter 
for the phenomenon is the normal state resistance of the junction. We find that by decreasing the damping in the system both the optimal power level and for maximum rectified voltage increase. The dependence of the bi-harmonic drive on the phenomenon can be qualitatively undertstood in a simple way. In the limit of the small rf frequency $f$ (with respect to the plasma frequency $f_{p}$ ) the kink behaves as a point-like particle and the system reacts to the rf drive adiabatically. For any chosen fundamental period of the microwave, the phase shift between two drives makes the total current large and positive over short time and then smaller and negative over somewhat longer time. Since the current amplitude is large enough to reach the saturation voltage for both current polarities (Swihart velocity in the case of fluxon), the mean voltage will be negative in this case. Changing the phase shift simply reverses the polarities of the currents and voltage. For higher frequencies, however, the adiabatic approach does not work and the phenomenon becomes more complicated. In particular, besides the point particle contribution characterizing the adiabatic regime, there is an equally important contribution to the drift current coming from internal oscillations of the kink (internal mode mechanism). These oscillations are asymmetric in space and phase locked to the external driver and, in presence of damping, they are coupled to the center of mass (translational mode) giving an extra contribution to the transport ${ }^{16}$. It would be interesting to separate these two contributions, a task which is not easy to perform within our present experimental setting.

In conclusion, we reported the first experimental observation of the rectified dc voltage, induced by the ratchet-like motion of a fluxon in uniform annular Josephson junction driven by bi-harmonic microwave fields. We find that the symmetry breaking in the rf field $\tilde{\gamma}(t)$ leads to the unidirectional motion of a fluxon, which is manifested in the non-zero rectified voltage across the junction at zero bias current. Our results qualitatively confirm the theoretical predictions made in Refs. 15, 16. The rectified voltage changes periodically with the phase shift $\theta$. The authors acknowledge discussions with P. L. Christiansen, M. Fistul, S. Flach, and M. R. Samuelsen. M. S. acknowledges partial support from a MURST-PRIN-2003 Initiative, and from the European grant LOCNET no. HPRN-CT-1999-00163. Y.Z. wishes to acknowledge support from the INTAS Young Scientist Fellowship no. 03-55-1799.

${ }^{1}$ A. Barone and G. Paternó, Physics and Applications of the Josephson Effect (Wiley: New York, 1982).

${ }^{2}$ A.V. Ustinov, Physica D 123, 315 (1998).

${ }^{3}$ G. Filatrella, B.A. Malomed, and R.D. Parmentier, Phys. Lett. A 198, 43 (1995).

${ }^{4}$ A. V. Ustinov and B. A. Malomed, Phys. Rev. B 64, 020302 (2001).

${ }^{5}$ E. Goldobin, B. A. Malomed and A. V. Ustinov, Phys. Rev. E 65, 056613 (2002).

${ }^{6}$ P. Hänggi and R. Bartussek, in: Nonlinear Physics of Complex Systems - Current Status and Future Trends, J. Parisi, S. C. Müller, W. Zimmermann (Eds), Lecture notes in physics 476, p.294 (Springer, Berlin 1996); F. Jülicher, A. Ajdari and J. Prost Rev. Mod. Phys., 69 (1997) 1269; P. Reimann, Phys. Rep. 361 (2002) 57.

${ }^{7}$ S. Flach, O. Yevtushenko and Y. Zolotaryuk, Phys. Rev. Lett. 84, 2358 (2000); O. Yevtushenko, S. Flach, Y. Zolotaryuk and A. A. Ovchinnikov, Europhys. Lett. 54, (2001) 141.

${ }^{8}$ M. Barbi and M. Salerno, Phys. Rev. E 63, 066212 (2001).

${ }^{9}$ E. Goldobin, A. Sterk, and D. Koelle, Phys. Rev. E 63, 031111 (2001).

${ }^{10}$ G. Carapella and G. Costabile, Phys. Rev. Lett. 87, 077002 (2001).

${ }^{11}$ F. Falo, P.J. Mart?nez, J.J. Mazo, and S. Cilla, Europhys. Lett. 45, 700 (1999).

12 E. Trias, J. J. Mazo, F. Falo, and T. P. Orlando, Phys. Rev. E 61, 2257 (2000).

13 J. B. Majer, J. Peguiron, M. Grifoni, M. Tusveld, and J. E. Mooij, Phys. Rev. Lett. 90, 056802 (2003).

${ }^{14}$ M. Salerno and N. Quinteiro, Phys. Rev. E 65, 025602(R) (2002).

15 S. Flach, Y. Zolotaryuk, A. E. Miroshnichenko, and M. V. Fistul, Phys. Rev. Lett. 88, 184101 (2002).

${ }^{16}$ M. Salerno and Y. Zolotaryuk, Phys. Rev. E 65, 056603 (2002).

${ }^{17}$ Using our layout, the samples were fabricated at the foundry of HYPRES Inc., Elmsford, NY 10523.

18 A. Davidson, B. Dueholm, B. Kryger, and N.F. Pedersen, Phys. Rev. Lett. 55, 2059 (1985).

19 N. Martuciello, J. Mygind, V.P. Koshelets, A.V. Schukin, L.V. Filippenko, and R. Monaco, Phys. Rev. B 57, 5444 (1998).

${ }^{20}$ A.V. Ustinov, B.A. Malomed, and N. Thyssen, Phys. Lett. A 233, 239 (1997).

${ }^{21}$ D. W. McLaughlin and A. C. Scott, Phys. Rev. A, 18 (1978) 1652; M. Salerno and A. C. Scott, Phys. Rev. B, 26 (1982) 2474; O. H. Olsen and M. R. Samuelsen, Phys. Rev. B, 28 (1983) 210. 


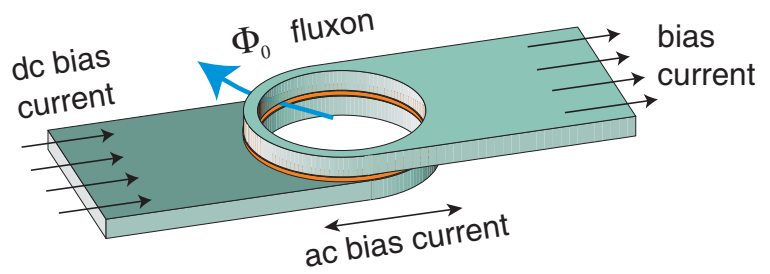

FIG. 1. Schematic view of an annular Josephson junction with a single fluxon trapped in it. The junction is biased by a dc current and an ac bias, induced by applied microwaves.
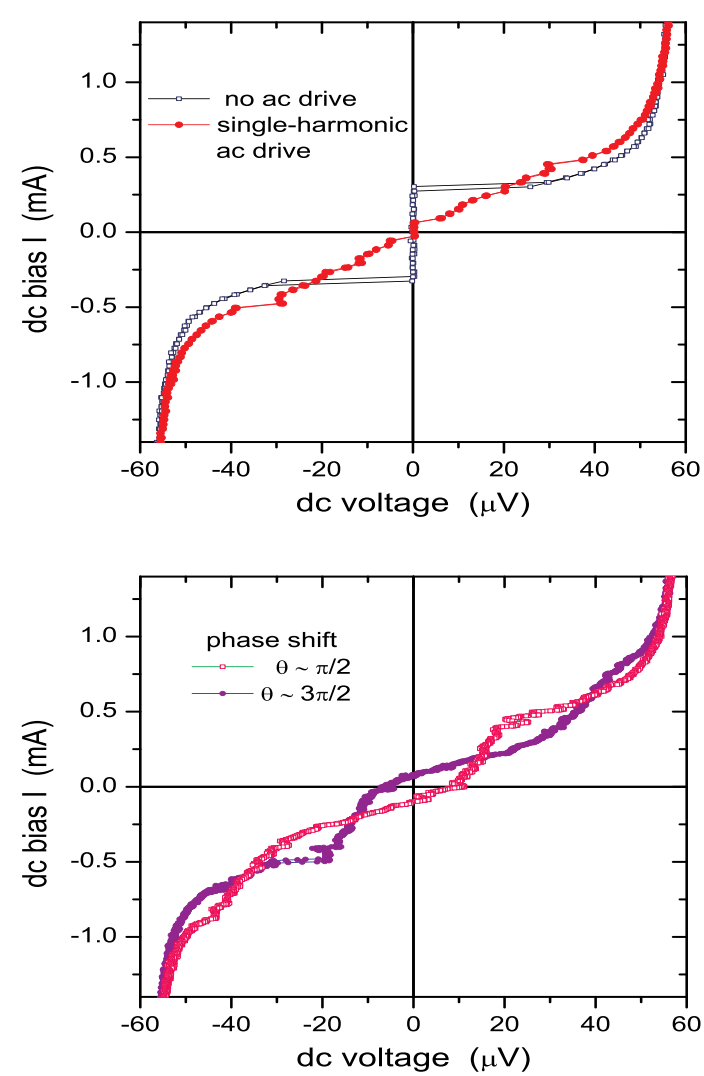

FIG. 2. (a) Current-voltage characteristics of a single fluxon in annular Josephson junction with no ac drive (open symbols) and with single-harmonic ac drive (solid symbols) having the frequency $f_{1}=4.8 \mathrm{GHz}$. (b) A single-fluxon current-voltage characteristics. The fluxon is driven by bi-harmonic ac drive having the frequencies $f_{1}=1.2 \mathrm{GHz}$ and $f_{2}=2.4 \mathrm{GHz}$ and power $P_{2}=+4 \mathrm{~dB}$. The phase shift $\theta$ between the two harmonics is fixed to $\pi / 2$ (open symbols) and $3 \pi / 2$ (solid symbols). 

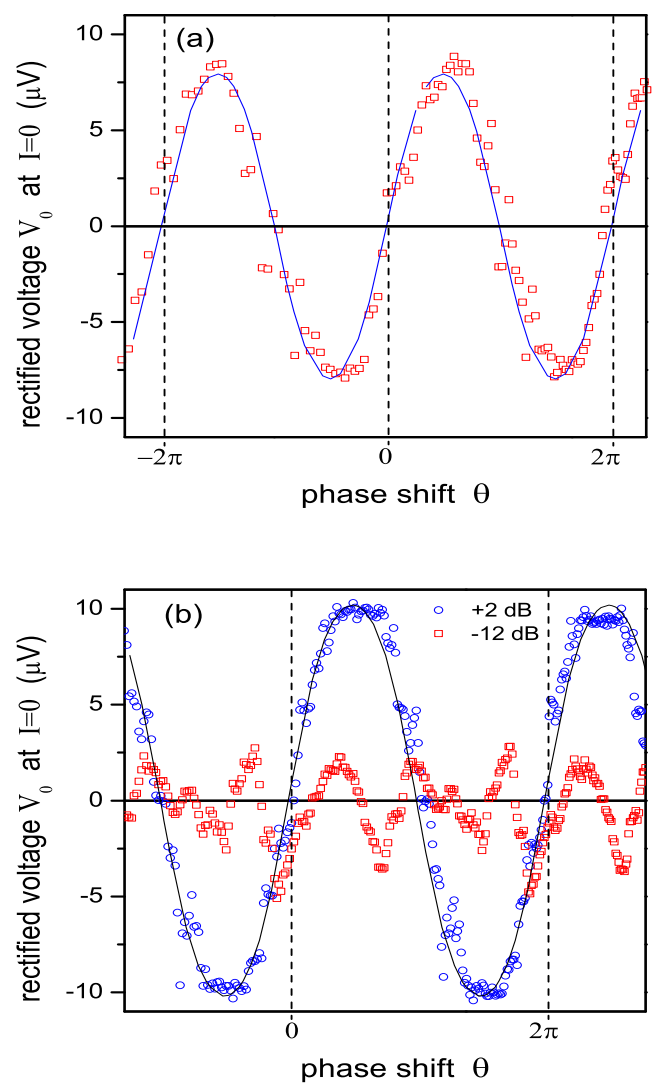

FIG. 3. A dependence of the rectified dc voltage measured at zero dc bias current on the phase shift $\theta$ between the two harmonics of ac drive. (a) $f_{1}=1.2 \mathrm{GHz}$ and $f_{2}=2.4 \mathrm{GHz}$ and power $P_{2}=+4 \mathrm{~dB}$. (b) $f_{1}=2.4 \mathrm{GHz}$ and $f_{2}=4.8 \mathrm{GHz}$, for two power levels. Solid lines show results of numerical computation of the voltage for the dimensionless ac bias amplitudes $E_{1}=E_{2}=0.1$ (a) and $E_{1}=E_{2}=0.135$ (b).

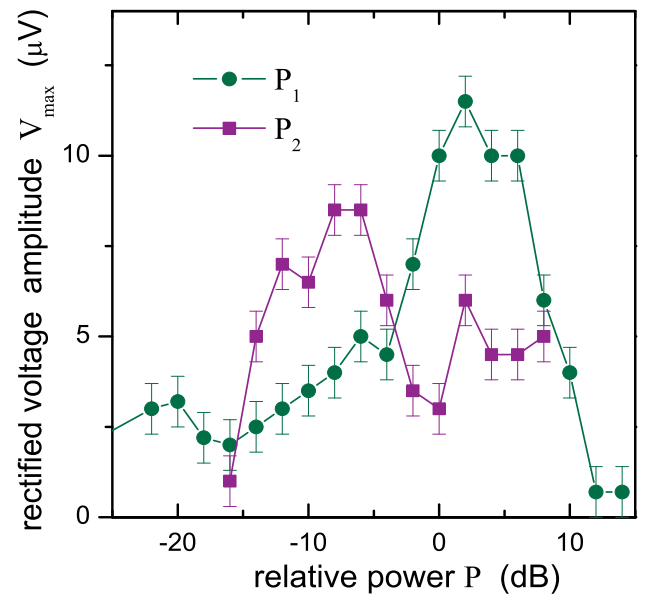

FIG. 4. A dependence of the maximum amplitude of the rectified dc voltage versus power for the two harmonics of ac drive. 